\title{
Lessons learned from China's mitigation strategies in fighting COVID-19
}

\author{
Sijiang Liu ${ }^{1, a, *, \dagger}$, Mingyuan Wan ${ }^{2, b, *, \dagger}$ \\ ${ }^{1}$ Department of Pharmaceutical Sciences, China Medical University-The Queen's University of Belfast Joint College, China Medical \\ University, 77 Puhe Road, Shenyang, 110122, China \\ ${ }^{2}$ Biochemistry, Biochemistry \& biomedical science, McMaster, L8S4L8, Canada \\ ${ }^{\dagger}$ These authors contributed equally.
}

\begin{abstract}
In late 2019, the first SARS-CoV-2 case was reported in Wuhan, China. It has been known as a deadly virus that could cause many severe health complications, particularly respiratory illnesses. With its extraordinary ability to transmit between humans, the coronavirus disease 2019 (COVID-19) has spread worldwide, including Antarctica and the Arctic region. On 11th March 2020, the World Health Organization (WHO) declared the COVID-19 as a public health emergency worldwide (global pandemic) to raise global awareness of the dangerous virus. With immediate and efficient public health interventions, progress has been seen in many countries such as China and New Zealand. Therefore, in this review, we summarized the important public health risk mitigation measures applied in China.
\end{abstract}

\section{Introduction}

According to WHO, "A pandemic is defined as an epidemic occurring worldwide, or over a very wide area, crossing international boundaries and usually affecting a large number of people" [1]. A number of pandemics that happened over human history, including smallpox, influenza, and tuberculosis. However, the one that affects us the most recently is the COVID-19 pandemic. Severe acute respiratory syndrome coronavirus 2 (SARS-CoV-2) is a positive-sense single-stranded RNA virus [1]. Its high genetic similarity with other coronavirus supports the idea that SARS-CoV-2 originates from bats [2]. WHO was informed about the COVID-19 outbreak in Wu Han, Hubei Province, China. However, a recent molecular genomics study suggested that the first case could be as early as November 2019 [3] and began to spread worldwide. International transmission started to accelerate since then. On 11 March 2020, the WHO officially characterized the COVID-19 outbreak as a pandemic. Up to this date, according to the real-time statistic from Johns Hopkins University, there are in total more than one billion confirmed cases worldwide and more than 2.5 million lives lost consisting of people of a wide range of ages [4]. Among all the infected regions, the U.S., Brazil, and India have the most cases, although the situation in Europe is not promising either ${ }^{4}$. While the cases increase dramatically for the rest of the world, the spread in China has been effectively controlled with approximately 100 thousand recorded cases overall [4]. This is the result of efficient public health interventions and risk mitigation strategies applied by the Chinese government. Thus, in this report, we summarized significant public health risk mitigation measures for the pre-, spark, and spread period of the pandemic in China and essential lessons that could be studied and utilized by other countries to combat COVID-19.

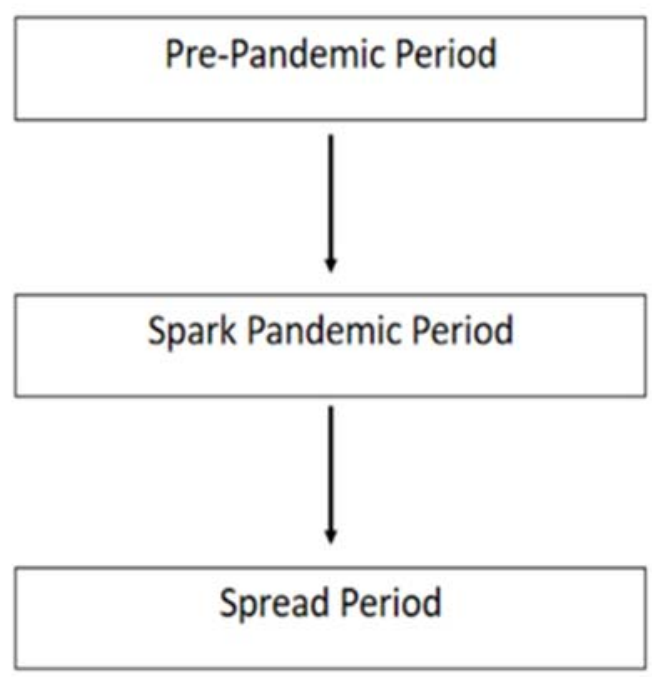

Figure1: the 3 three stages of the pandemic

\section{Pre-pandemic Period}

Over the past few decades, due to the careful implementation of the health policy, China's health work has made great progress and achieved remarkable

*Corresponding author. Email:a sliu23@qub.ac.uk, ${ }^{b}$ w602146211@gmail.com 
achievements. In the early $60 \mathrm{~s}$, China was the first declared smallpox eradication, ahead of the world of smallpox extinction for ten years. It gradually eliminates the classical biotype cholera, plague, relapsing fever, kala-azar, serious harm people's health of infectious diseases such as typhus and plans in 1995 wild strains of paralytic polio [5]. Many endemic diseases have been basically controlled.

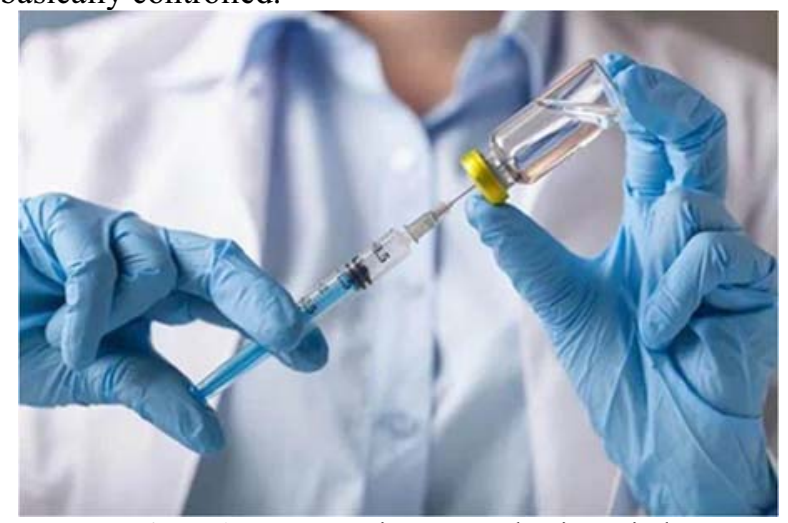

Figure2: treatment in pre-pandemic period

The incidence of infectious diseases in China has decreased remarkably. Due to carrying out a series of regulations, health standards, and management measures, such as food hygiene law, the prevention and control of infectious diseases, maternal and infant health care law, regulations on pneumoconiosis prevention, school health workers, the industrial and mining working conditions gradually improved, could improve the health of primary and middle school students, are obtained by the medical, learn food safety and health education network, and further to ensure the health of the population. China's health institutions at all levels have also made great progress.A nationwide urban and rural health service system, a three-level network for medical prevention and health care, has been basically formed. A health team has begun to take shape, ensuring the basic medical conditions for the vast number of residents. To provide primary health care services to the population, a threelevel medical prevention and health care network was established. It includes county-level general hospitals, health centers, and village clinics for rural residents, neighborhood, district, and city hospitals for urban residents, aiming to provide medical, prevention, health care, and other health tasks.

As a result, the health of the Chinese people has been continuously improved. The national mortality rate and infant mortality have dropped obviously. The population's average life expectancy was 35 years before founding the People's Republic of China [9]. At present, China's overall economic level is still under-developed, and it belongs to a developing country. However, some important health indicators of its residents, such as birth rate, death rate, and life expectancy, have surpassed other developing countries and are higher than the world average. Some of them are close to the level of developed countries. All these are achievements of China's health work.

\section{Spark period}

\subsection{Contain transmission}

In the initial phase of an outbreak, the "early case identification with large-scale surveillance" intervention is essential in containing the spread of the disease as no drugs or vaccines have yet been developed [6]. At the start of the COVID-19 outbreak, most theories postulated that the Huanan seafood market in Wuhan is the origin where the virus was firstly introduced [7]. Therefore, Chinese authorities started their surveillance by investigating multiple food markets to look for the infection source ${ }^{6}$. Meanwhile, to identify the potentially infected individuals, the national authorities hired 1800 teams of epidemiologists and assigned them to trace tens of thousands of people per day [7]. Big data is frequently used in screening procedures ${ }^{8}$. Residents and travelers were requested to have their temperature checked and presented with green health (Quick Response Code) QR code if the individual is identified as low transmission risk based on previous travel history [8]. Only people who possess these QR codes could travel to work, school, and public facilities. In addition, the Chinese government uses the street camera system equipped with a facial recognition program to track down individuals and evaluate transmission risk [7]. Later, fast COVID-19 testing techniques were developed, and China made the tests available and accessible for every individual. COVID-19 testing was mandatory for every residence in the high-risk community, and people who tested positive will be forced to be isolated [8]. Also, assigned individuals will be sent off to reinforce that the residents follow the isolation rules and check their health status.

\subsection{Risk communication}

Risk communication is also critical in the initial phase of a disease outbreak. To actively communicate and educate the public about the severity of the disease and the importance of the mitigation strategies, the Chinese government utilized many social media to share the information, including WeChat, TikTok, Kwai, Bilibili, and Weibo [8]. Basic knowledge of the virus and practical prevention methods such as wearing masks, washing hands, and sanitizing the container of groceries, was advertised. Radio, broadcast television, Newspaper, and billboards are also used in risk communication for older generations who do not access the new media frequently [8]. The telephone hotline 12345 is available in eight languages, and the English service is available 24 hours for foreign residents in cities like Shanghai and Beijing [8]. In addition, data and information were publicized and updated hourly on many websites to strengthen the public awareness of the virus. The practical education about the seriousness of the disease made people trust and support the government's decision-making process and cooperate with the complete lockdown policy later on in the combat with the COVID-19 pandemic. 


\section{Spread Period}

\subsection{International cooperation}

The Chinese government has done a good job in information dissemination and international cooperation. They release timely, open, and transparent information on the epidemic, objectively report the progress of the epidemic and the prevention and control measures taken by the government, and scientifically disseminate knowledge on prevention and control of the epidemic. China continues to take the initiative to strengthen communication with the World Health Organization (WHO) on the epidemic, promptly share information on epidemic surveillance, investigation, prevention, and control, as well as opinions on risk assessment, and jointly discuss and improve epidemic prevention and control measures.

\subsection{Efforts of the healthcare system}

Public health workers are resolutely implementing epidemic prevention and control measures, ensuring continuous and improved surveillance for pneumonia of unknown cause and pre-testing and triage in fever outpatient clinics, making all preparations for case treatment, and promptly detecting and effectively dealing with the epidemic [9].

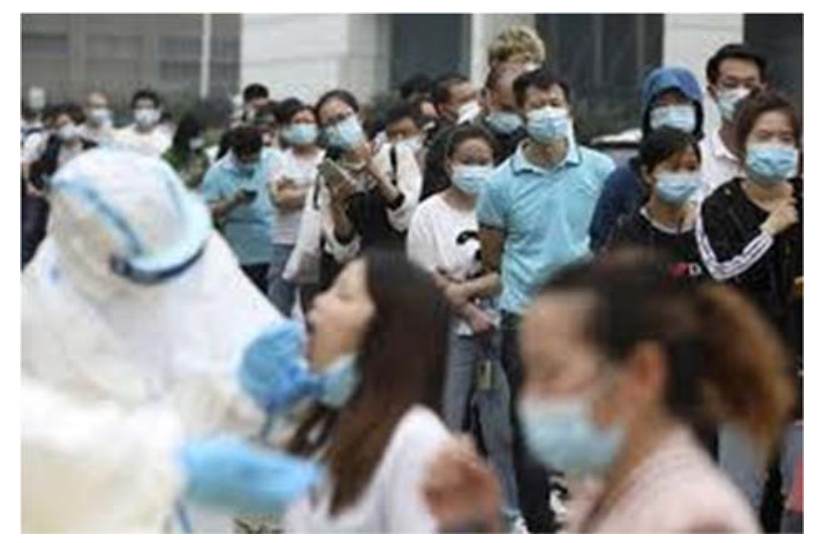

Figure3: Public health workers are taking nucleic acid detection

The hospital has made every effort to treat the patients by deploying the strongest Chinese and Western medical resources and expert resources and combining traditional Chinese and Western medicine to minimize the number of deaths, strengthen the guarantee of medical treatment costs for patients and ensure timely treatment for patients [10]. The government takes care of medical staff, provides logistical support, arranges rest reasonably, and especially instructs them to take good personal protection to prevent them from being infected in treating patients.

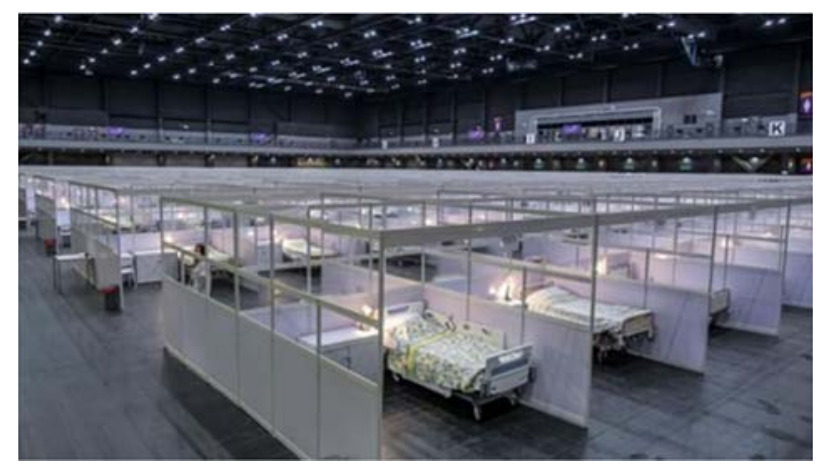

Figure4: Fangzhou for COVID-19

Experts step up scientific research on epidemic prevention and control, give full play to experts, identify the source of infection and transmission routes as soon as possible. They research, develop and screen antiviral drugs in a targeted way, closely track and monitor changes in virulence and transmission of the virus, and make epidemic prevention and control more scientific and effective.

\subsection{Vaccine}

On August 5, 2020, the production workshop of COVIDinactivated vaccine in Beijing Institute of Biological Products affiliated to Sino pharmaceutical China Institute of Biology passed the joint biosafety inspection organized by relevant national departments and was qualified for use. Previously, the workshop had obtained a COVID-19 vaccine production license; On August 16, Academician Chen Wei's team obtained a patent for the new coronavirus vaccine, the first of its kind in China [11]. The patent application for this invention enjoys a preferential review policy. On August 20th, national medicine group, China's biological inactivated vaccine clinical trials of the new champions league start in Peru (III period); On October 8, China signed an agreement with the Global Alliance for Vaccines and Immunization (GAVI) to officially join the COVID-19 Vaccine Implementation Plan. As of October 20, 2020, China has vaccinated about 60,000 subjects, and no serious adverse reactions have been reported [12]. As of February 25, 2021 , four new coronavirus vaccines have been listed in China, including three inactivated vaccines and one adenovirus vector vaccine. 


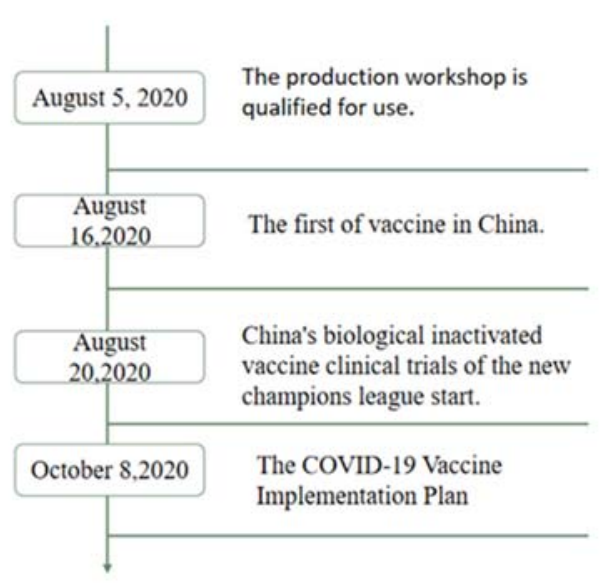

Figure5: The R\&D process of vaccine

Most methods are applied effectively and helpful. They can be effective suggestions for other countries such as releasing timely, open, and transparent information on the epidemic, the high executive force of hospital and public health organization, fully playing the role of the expert, developing safe and effective vaccines rapidly [13].

\section{Valuable lessons}

Many of these mitigation methods mentioned above helped China successfully monitor the infection trend and ensure the control of the spread of the disease. Therefore, valuable experience can be studied, and similar approaches could be applied in other countries. For example, governments could use big data to analyze individuals' travel history and provide some sort of identification documents once they are determined to have low transmission risk. Then impose restrictions towards unessential travel for people who do not possess these documents. In addition, for people who tested positive, the government could establish communitywide organizations to constantly monitor the local positive cases and check on these individuals to make sure they follow the isolation rules strictly. During the spread period, it is necessary to establish an efficient communication system to disseminate information and encourage international cooperation. Public health systems need to assist each other in fighting against diseases and viruses. In addition, developing vaccines is also necessary. Some of these interventions are rigorous, but they provide a public health system with greater surveillance capacities during a challenging situation. Also, with China's success in fighting the pandemic as evidence, these mitigation methods should indeed be considered or studied by other countries.

\section{Conclusion}

In the pre-pandemic period, China had improved some important health indicators of its residents and surpassed other developing countries and are higher than the world average. Some of them are close to the level of developed countries. Some effective methods are used in the spark period, such as green health QR codes, COVID-19 testing. Vaccine development continues during the spread period, and four new coronavirus vaccines have been listed in China, including three inactivated vaccines and one adenovirus vector vaccine. Many of the above countermeasures for COVID-19 have helped China successfully monitor infection trends and ensure that the spread of the disease is controlled. These valuable lessons can therefore be studied and similar approaches applied to other countries.

\section{Reference}

1. WHO | The Classical Definition of a Pandemic Is Not Elusive. 2011

2. Hu, B.; Guo, H.; Zhou, P.; Shi, Z.-L. Characteristics of SARS-CoV-2 and COVID-19. Nature Reviews Microbiology 2020, 19 (3), 141-154.

3. Parczewski, M.; Ciechanowicz, A. Molecular Epidemiology of SARS-CoV-2: A Review of Current Data on Genetic Variability of the Virus. Pol. Arch. Intern. Med. 2020, 131 (1), 63-69.

4. COVID-19 map - johns Hopkins Coronavirus resource Center https://coronavirus.jhu.edu/ map.html (accessed Mar 18, 2021).

5. Wang L, Wang Z, Ma Q, Fang G, Yang J. The development and reform of public health in China from 1949 to 2019. Global Health. 2019;15(1):45.

6. Yu, X.-Y.; Xu, C.; Wang, H.-W.; Chang, R.-J.; Dong, Y.-Q.; Tsamlag, L.; Zhang, S.-X.; Yu, Y.-L.; Long, R.-S.; Wang, H.; Cai, Y. Effective Mitigation Strategy in Early Stage of COVID-19 Pandemic in China. Infect. Dis. Poverty 2020, 9 (1), 141.

7. AlTakarli, N. S. China's Response to the COVID-19 Outbreak: A Model for Epidemic Preparedness and Management. Dubai Med. J. 2020, 3 (2), 44-49.

8. Jiang, B.; Li, W.; Yuan, T. Coronavirus Disease 2019: Community Risk Mitigation Strategies in China. J. Emerg. Manag. Disaster Commun. 2020, 01 (01), 73-87.

9. Ye Q, Wang B, Mao J, Fu J, Shang S, Shu Q, et al. Epidemiological analysis of COVID-19 and practical experience from China. J Med Virol. 2020;92(7):755-69.

10. Tang YW, Schmitz JE, Persing DH, Stratton CW. Laboratory Diagnosis of COVID-19: Current Issues and Challenges. J Clin Microbiol. 2020;58(6).

11. Hu B, Guo H, Zhou P, Shi ZL. Characteristics of SARS-CoV-2 and COVID-19. Nat Rev Microbiol. 2021;19(3):141-54.

12. Sharma O, Sultan AA, Ding H, Triggle CR. A Review of the Progress and Challenges of Developing a Vaccine for COVID-19. Front Immunol. 2020;11:585354.

13. Amawi H, Abu Deiab GI, AA AA, Dua K, Tambuwala MM. COVID-19 pandemic: an overview of epidemiology, pathogenesis, diagnostics and potential vaccines and therapeutics. Ther Deliv. 2020;11(4):245-68. 